\title{
Non-alcoholic fatty liver disease
}

\author{
Authors: Wenhao $\mathrm{Li}^{\mathrm{A}}$ and William Alazawi ${ }^{\mathrm{B}}$
}

\begin{abstract}
Non-alcoholic fatty liver disease (NAFLD) is the most common cause of chronic liver disease worldwide. NAFLD is defined by excess fat in the liver and has a multidirectional relationship with metabolic syndrome. The prevalence of NAFLD has risen rapidly in recent years in line with the obesity epidemic and associated increases in type 2 diabetes, hypertension and hypercholesterolaemia. Patients with NAFLD are at risk of cardiovascular disease and cancer, and in a proportion of individuals, NAFLD is associated with liver damage. This article summarises the epidemiology of NAFLD, the clinical approach to risk-assessing patients and briefly outlines current and future management options.
\end{abstract}

\section{Introduction}

Non-alcoholic fatty liver disease (NAFLD) is defined as the histological or radiological presence of excess fat in more than $5 \%$ of hepatocytes (steatosis) in the absence of secondary causes. These include alcohol excess, drugs (such as amiodarone, valproate or methotrexate), viral hepatitis, endocrine abnormalities (such as polycystic ovarian syndrome) and glycogen storage disorders. Recently, a consensus statement has proposed a change in nomenclature to metabolic-associated fatty liver disease although this has yet to be universally accepted. ${ }^{1}$

\section{Epidemiology and natural history}

Estimates of prevalence of NAFLD have been based on cohort studies and by extrapolation from known rates of diabetes and obesity. In the general population, prevalence varies between $13-32 \%$ globally and is approximately $25 \%$ in the west (Fig 1). ${ }^{2-4}$ NAFLD affects $60-80 \%$ among people living with type 2 diabetes. ${ }^{5}$ However, prevalence rates of recorded diagnoses of NAFLD in realworld primary care datasets are much lower than these, reflecting large numbers of undiagnosed patients and perhaps scope for improved understanding of NAFLD and its significance.

Not all patients with NAFLD progress to advanced liver disease. Up to one-third of people with NAFLD can develop the progressive fibroinflammatory form - non-alcoholic steatohepatitis (NASH). ${ }^{6}$ $\mathrm{NASH}$ is diagnosed by liver biopsy and reported according to semi-quantitative assessment of steatosis, lobular inflammation,

Authors: ${ }^{A}$ hepatology research fellow, Barts Liver Centre, London,

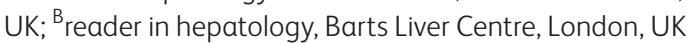

hepatocyte ballooning and fibrosis. The presence of NASH is a key driver for the development of fibrosis with data indicating that fibrosis progresses twice as fast in patients with NASH compared to those without. Nevertheless, it is the extent of fibrosis (stage) rather than the degree of ballooning or inflammation (grade) that predicts life-threatening outcomes such as cirrhosis, liver failure, liver cancer and mortality. ${ }^{7}$ Therefore, a key clinical objective is to identify those patients with fibrosis due to NASH who may be at risk of significant liver disease.

The pathogenesis of NASH is complex and involves the interaction of multiple 'hits'. Age, sex and ethnicity all play a role, as does genetic predisposition with much focus on PNPLA3 and TM6SF2 genes among others. The inflammatory triggers in NASH probably relate to lipid-mediated toxicity within hepatocytes as well as the effect of the altered gut microbiome. The strongest risk factors for NASH and fibrosis are type 2 diabetes and related metabolic disorders including obesity. Paired biopsy and population studies indicate that in patients with established $\mathrm{NASH}$, those with diabetes are at greatest risk of progression to end-stage liver disease and cancer. ${ }^{8,9}$

\section{Key points}

Non-alcoholic fatty liver disease (NAFLD) is the most common cause of chronic liver disease worldwide.

The gold standard for diagnosis of non-alcoholic steatohepatitis (NASH) and fibrosis is liver biopsy.

Non-invasive tests (Fibrosis-4 (FIB-4) index for liver fibrosis, NAFLD fibrosis score, enhanced liver fibrosis score and transient elastography) can help to identify patients at risk of significant liver fibrosis.

Stage of liver fibrosis rather than grade of NASH predicts liver-related outcomes.

Behavioural and lifestyle modifications remain the cornerstone of treatment as there are currently no licensed medications for $\mathrm{NASH}$ in Europe.

KEYWORDS: Non-alcoholic fatty liver disease, non-alcoholic steatohepatitis, fibrosis, cirrhosis

DOI: 10.7861/clinmed.2020-0696 
Fig 1. Non-alcoholic fatty liver disease spectrum. Approximate estimates of prevalence in the general population (black text) and rates of progression or regression (teal text and arrows). ${ }^{3,4}$ $\mathrm{NASH}=$ non-alcoholic steatohepatitis.
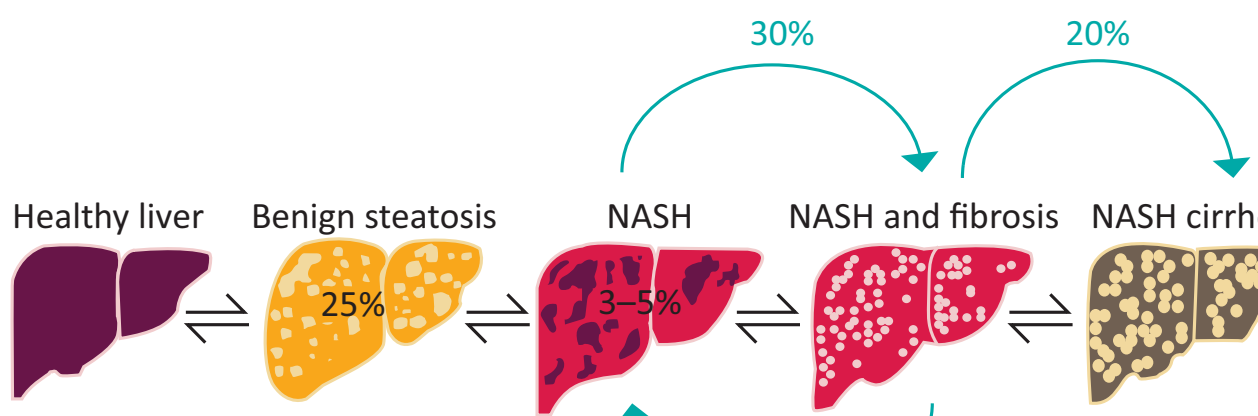
NASH

NASH and fibrosis

NASH cirrhosis
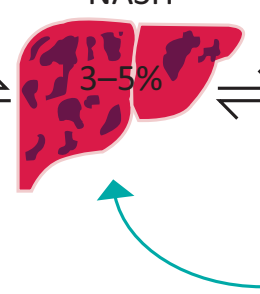

$20 \%$

\section{Diagnosis and risk stratification}

Excess liver fat is usually determined by ultrasound, which may be performed for various reasons: eg investigating abnormal liver biochemistry or unrelated abdominal symptoms. Clinical assessment or serum transaminase levels alone are inadequate for detecting fibrosis and identifying which patients will have a benign versus progressive disease course. Indeed, patients at all stages of disease can have normal transaminases. Quality of life can be significantly impaired at all stages of NAFLD but, for most people, there are no specific symptoms and so many are diagnosed at late stages of disease with cirrhosis, liver failure or cancer. ${ }^{10}$

NASH and fibrosis cannot be reliably and accurately diagnosed without a liver biopsy, but it is neither feasible nor desirable to biopsy everyone with NAFLD because it is an invasive technique with associated costs and morbidity. However, there are a number of non-invasive tests that are calculated from routinely collected clinical blood results and that can give a proxy estimate of the risk of fibrosis in patients with a clinical diagnosis of NAFLD, including Fibrosis-4 (FIB-4) index for liver fibrosis and the NAFLD fibrosis score (NFS). ${ }^{11}$ The necessary laboratory results (alanine aminotransferase (ALT), aspartate transaminase (AST) and platelet count) must be available, but most biochemistry laboratories will provide one of ALT or AST but not both unless specifically requested. In the UK's The Health Improvement Network's (THIN) primary care database, only $14 \%$ of patients with a recorded diagnosis of NAFLD have had the laboratory tests performed to calculate the simplest of these scores, and it is not known whether scores were actually calculated. ${ }^{12}$ Non-invasive test scores correlate well with liver outcomes, have high specificity with areas under receiver-operator curves for advanced fibrosis over $0.8 .^{13,14}$ National and international guidance, including National Institute for Health and Care Excellence (NICE) guideline NG49, recommend the use of non-invasive tests of fibrosis and as a screening tool to identify patients who are likely to have progressive liver disease. ${ }^{15-17}$ Most experts would recommend a two-tier approach with FIB-4 or NFS ruling out patients very unlikely to have significant fibrosis, followed by a second test for those with indeterminate or high-risk scores (an example of such a pathway published by the British Society of Gastroenterology is presented in Fig 2). ${ }^{18}$ The second tier usually tests for a biomarker of fibrosis (eg enhanced liver fibrosis (ELF) score) or transient elastography (eg FibroScan) and patients with evidence of fibrosis are referred to secondary care for further assessment, and considered for existing and emerging therapies. ${ }^{19}$

\section{Existing and emerging treatments}

The cornerstone of managing NAFLD is behaviour and lifestyle change, focusing on weight loss, dietary modification and increase in physical exercise which should be offered to all patients, irrespective of whether there is evidence of NASH and fibrosis. Similarly, optimisation of cardiovascular risk factors including hypercholesterolaemia, hypertension and diabetes should follow existing NICE guidance (CG181, NG136 and NG28). ${ }^{20-22}$

Intensive lifestyle change can lead to improvements in serum transaminases and markers of insulin resistance even without weight loss. Histological endpoints of NASH resolution and improvement in fibrosis can be achieved with lifestyle-induced weight loss and are most frequently seen in those who lose $>7 \%$ of starting body weight. ${ }^{23}$ However, few can achieve and maintain this level of weight reduction through lifestyle measures alone. There are not nationally agreed standards on the composition of lifestyle interventions for obesity in general or NASH in particular, leading to wide regional disparities in these services. Bariatric or metabolic surgery has emerged in recent years as a safe and effective therapy and can result in dramatic weight loss, most of which is maintained. Bariatric surgery can lead to resolution of diabetes, improvement in cardiometabolic risk factors and in case-series, improvement in liver histology, although randomised controlled diet data are currently lacking. NICE guidance suggests considering treatment with vitamin E or pioglitazone for patients with NASH, based largely on the results of the PIVENS study which showed improvement in NASH (primary endpoint) for vitamin $\mathrm{E}$ and resolution of NASH (secondary endpoint) for pioglitazone with neither having a significant impact on fibrosis after 96 weeks' treatment. ${ }^{24}$ Concerns regarding the safety of both drugs as well as the impact of reducing NASH without affecting fibrosis are the subject of ongoing debate.

New classes of drugs are emerging for the treatment of NASH and fibrosis, and existing drugs are undergoing repurposing studies. Agonists of the farnesoid X receptor (FXR), a nuclear hormone receptor that regulates bile acid metabolism, protect against liver inflammation and fibrosis in a murine model of 


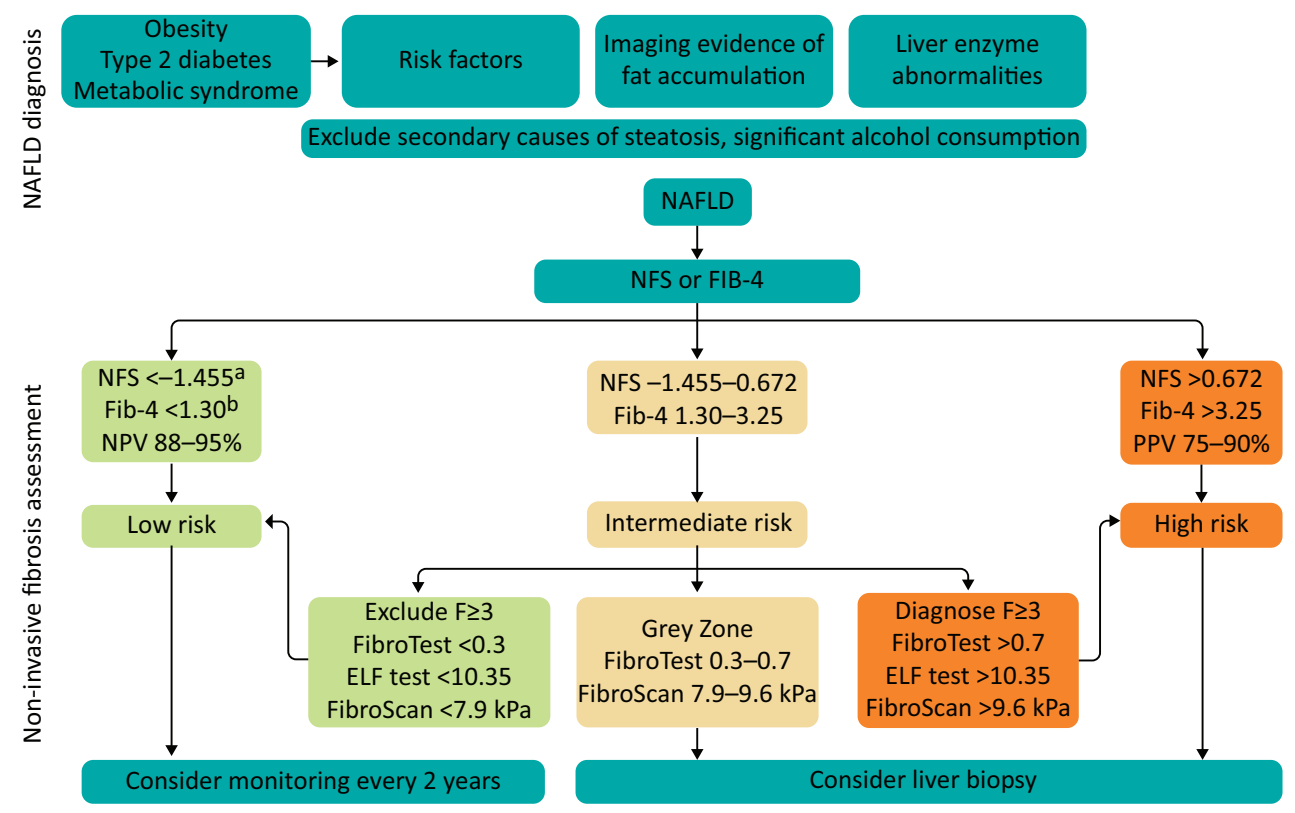

Fig 2. Proposed algorithm for diagnosis of non-alcoholic fatty liver disease and noninvasive assessment of liver fibrosis. Adapted from Horn P, Newsome PN. NAFLD - diagnosis, assessment and management. British Society of Gastroenterology, $2020 .^{18 a}=$ NFS threshold $<0.12$ for patients $>65$ years; ${ }^{b}=$ FIB-4 threshold $<2.0$ for patients $>65$ years old; ELF $=$ enhanced liver fibrosis score; FIB-4 $=$ Fibrosis- 4 index for liver fibrosis; NAFLD = non-alcoholic fatty liver disease; NFS = NAFLD fibrosis score; $N P V=$ negative predictive value; $P P V=$ positive predictive value.

NASH. ${ }^{25}$ This class of drugs probably has the largest body of evidence in NASH to date with interim results from a trial of obeticholic acid (already licenced for the treatment of primary biliary cholangitis) recently demonstrating some benefit over placebo. ${ }^{26}$ As a class, FXR agonists are associated with pruritus and appear to increase serum low-density lipoproteins. Glucagon-like peptide-1 (GLP-1) agonists improve insulin resistance and induce a modest degree of weight loss and are used in patients with type 2 diabetes, but are not licensed for the treatment of NASH or liver fibrosis. The LEAN study demonstrated that NASH resolution was significantly more frequently seen in patients treated with the GLP-1 receptor agonist liraglutide compared to those on placebo. ${ }^{27}$ This trial has led to trials of semaglutide and dulaglutide currently in phase III/IV trials. Thyroid hormone receptor-beta agonists such as resmetirom or VK2809 are currently undergoing trials as are cenicriviroc (inhibitor of CCL2/5 chemokines), and aramchol (stearoyl-CoA dehydrogenase-1 inhibitor) among many others. Over 40 drugs are currently in different phases of clinical trials and, although there is some variation in study design, the majority of advanced phase studies have clinical endpoints with histological assessment as an interim readout after 12 or 18 months.

Regardless of whether and when safe, efficacious and costeffective drugs become available, clinical services should focus on supporting individual behaviour and lifestyle change which will positively impact NAFLD, NASH, diabetes and cardiovascular risk. Services should be organised around patient need with access to specialist nursing, dietetics, physical exercise and psychological support. Clinicians can also play a role in advocating for making such services available and addressing the obesogenic environment which is doubtless contributing to the rise in NAFLD and other metabolic disorders.

\section{References}

1 Eslam M, Sanyal AJ, George J, International Consensus Panel. MAFLD: A consensus-driven proposed nomenclature for metabolic associated fatty liver disease. Gastroenterology 2020;158:1999_ 2014.e1

2 Friedman SL, Neuschwander-Tetri BA, Rinella M, Sanyal AJ. Mechanisms of NAFLD development and therapeutic strategies. Nat Med 2018;24:908-22.

3 Younossi ZM. Non-alcoholic fatty liver disease - A global public health perspective. J Hepatol 2019;70:531-44.

4 Loomba R, Adams LA. The $20 \%$ rule of NASH progression: The natural history of advanced fibrosis and cirrhosis caused by NASH. Hepatology 2019;70:1885-8.

5 Bril F, Cusi K. Nonalcoholic fatty liver disease. Endocrinol Metab Clin North Am 2016;45:765-81.

6 Williams CD, Stengel J, Asike MI et al. Prevalence of nonalcoholic fatty liver disease and nonalcoholic steatohepatitis among a largely middle-aged population utilizing ultrasound and liver biopsy: a prospective study. Gastroenterology 2011;140:124-31.

7 Hagström H, Nasr P, Ekstedt M et al. Fibrosis stage but not NASH predicts mortality and time to development of severe liver disease in biopsy-proven NAFLD. J Hepatol 2017;67:1265-73.

8 Alexander M, Loomis AK, van der Lei ] et al. Risks and clinical predictors of cirrhosis and hepatocellular carcinoma diagnoses in adults with diagnosed NAFLD: real-world study of 18 million patients in four European cohorts. BMC Med 2019;17:95.

9 McPherson S, Hardy T, Henderson E et al. Evidence of NAFLD progression from steatosis to fibrosing-steatohepatitis using paired biopsies: Implications for prognosis and clinical management. J Hepatol 2015;62:1148-55.

10 Huber Y, Boyle M, Hallsworth K et al. Health-related quality of life in nonalcoholic fatty liver disease associates with hepatic inflammation. Clin Gastroenterol Hepatol 2019;17:2085-92.e1.

11 Vallet-Pichard A, Mallet V, Nalpas B et al. FIB-4: An inexpensive and accurate marker of fibrosis in HCV infection. comparison with liver biopsy and fibrotest. Hepatology 2007;46:32-6.

12 Alexander M, Loomis AK, Fairburn-Beech J et al. Real-world data reveal a diagnostic gap in non-alcoholic fatty liver disease. BMC Med 2018;16:130.

13 Angulo P, Bugianesi E, Einar S Bjornsson ES et al. Simple noninvasive systems predict long-term outcomes of patients with nonalcoholic fatty liver disease. Gastroenterology 2013;145:782-9.e4. 
14 Xiao G, Zhu S, Xiao X et al. Comparison of laboratory tests, ultrasound, or magnetic resonance elastography to detect fibrosis in patients with nonalcoholic fatty liver disease: A meta-analysis. Hepatology 2017:66:1486-501.

15 European Association for the Study of the Liver (EASL), European Association for the Study of Diabetes (EASD), European Association for the Study of Obesity (EASO). EASL-EASD-EASO clinical practice guidelines for the management of non-alcoholic fatty liver disease. J Hepatol 2016;64:1388-402.

16 Newsome PN, Cramb R, Davison SM et al. Guidelines on the management of abnormal liver blood tests. Gut 2018;67:6-19.

17 National Institute for Health and Care Excellence. Non-alcoholic fatty liver diseaseNAFLD: assessment and management: NICE guideline [NG49]. NICE, 2016.

18 Horn P, Newsome PN. NAFLD - diagnosis, assessment and management. British Society of Gastroenterology, 2020. www.bsg.org.uk/ clinical-articles-list/nafld-diagnosis-assessment-and-management

19 Srivastava A, Gailer R, Tanwar S et al. Prospective evaluation of a primary care referral pathway for patients with non-alcoholic fatty liver disease. J Hepatol 2019;71:371-8.

20 National Institute for Health and Care Excellence. Cardiovascular disease: risk assessment and reduction, including lipid modification: Clinical guideline [CG181]. NICE, 2016.

21 National Institute for Health and Care Excellence. Hypertension in adults: diagnosis and management: NICE guideline [NG136]. NICE, 2019.
22 National Institute for Health and Care Excellence. Type 2 diabetes in adults: management: NICE guideline [NG28]. NICE, 2019.

23 Vilar-Gomez E, Martinez-Perez Y, Calzadilla-Bertot L et al. Weight loss through lifestyle modification significantly reduces features of nonalcoholic steatohepatitis. Gastroenterology 2015;149:367-78.e5.

24 Sanyal AJ, Chalasani N, Kowdley KV et al. Pioglitazone, vitamin E, or placebo for nonalcoholic steatohepatitis. N Engl ] Med 2010; 362:1675-85.

25 Goto T, Itoh M, Suganami T et al. Obeticholic acid protects against hepatocyte death and liver fibrosis in a murine model of nonalcoholic steatohepatitis. Sci Rep 2018;8:8157.

26 Younossi ZM, Ratziu V, Loomba R et al. Obeticholic acid for the treatment of non-alcoholic steatohepatitis: interim analysis from a multicentre, randomised, placebo-controlled phase 3 trial. Lancet 2019;394:2184-96.

27 Armstrong MJ, Gaunt P, Aithal GP et al. Liraglutide safety and efficacy in patients with non-alcoholic steatohepatitisLEAN: a multicentre, double-blind, randomised, placebo-controlled phase 2 study. Lancet 2016;387:679-90.

Address for correspondence: Dr William Alazawi, Barts Liver Centre, Blizard Institute, Queen Mary University of London, 4 Newark Street, London E1 2AT, UK.

Email: w.alazawi@qmul.ac.uk

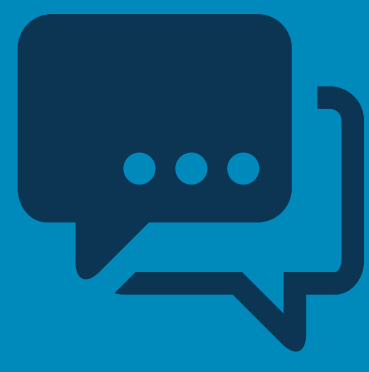

